Journal of The Magnetics Society of Japan Vol. 13, Supplement, No. S1 (1989) (C) 1989 by The Magnetics Society of Japan

\title{
GRAPHICAL ANALYSIS OF PERPENDICULAR MAGNETIC RECORDING CHARACTERISTICS
}

Kohsuke NISHIMURA, Yoshihisa NAKAMURA and Shun-ichi IWASAKI

Res. Inst. Elec. Comm., Tohoku Univ., 2-1-1, Katahira, Aoba-ku, Sendai 980, Japan

\begin{abstract}
In longitudinal magnetic recording on thin film media, the width of magnetization transition can be calculated graphically and quantitatively by taking the influence of demagnetizing field into account. This method can be also applied to perpendicular magnetic recording without taking this influence into account. Using the graphical metnod, not only isolated pulses but also bit density characteristics can be estimated quantitatively in good agreement with measured results.
\end{abstract}

\section{INTRODUCTION}

In perpendicular magnetic recording using a single pole head and a double layered medium, we can record and reproduce signals at an extremely high bit density more than $20 \mathrm{kFRPM} \mathrm{[1].} \mathrm{This} \mathrm{fact} \mathrm{shows}$ that the magnetization transition in perpendicular magnetic recording is considerably sharp in comparison with that in longitudinal magnetic recording because of no demagnetizing field effect.

In order to theoretically understand such a recording mechanism and to quantitatively investigate the optimum conditions for an ultra-high density recording system, we are pushing the computer simulation on both perpendicular and longitudinal magnetic recordings [2]. In this paper, however, we describe that the magnetization transition width can be also analysed approximately but quantitatively by a graphical method for both perpendicular and longitudinal magnetic recordings [3]. Moreover, we will discuss the fundamental differences between both recordings from the results.

\section{GRAPHTCAL ANALYSIS}

\section{Inherent Magnetization Transition}

At first, we calculate graphically an inherent magnetization transition width which is the width of an isolated magnetization transition given independently of the demagnetizing field effect. For the analysis, we assume the following things:

(1) The magnetization in a medium and the magnetic field of a head are one-dimentiona1ly treated by the only component in the head-moving direction for longitudinal magnetic recording or in the perpendicular direction to the medium surface for perpendicular recording.

(2) The strength of head field is constant through a magnetic layer in a thin film medium and so the mean field distribution which is average through the magnetic layer is used.

One-dimentional magnetization distribution around a transition region can be graphically obtained without taking the demagnetizing field effect into account as shown in Fig. 1(c) from the field distribution of a head (a) and the residual magnetization curve (b) of a medium. The inherent transition width, a", therefore, can be obtained from the slope at the, center of the transition as shown in Fig. 1(c).

If residual magnetization, $M_{r}$, of a medium becomes $\mathrm{O}$ at field strength, $\mathrm{H}_{\mathrm{rc}}$, as

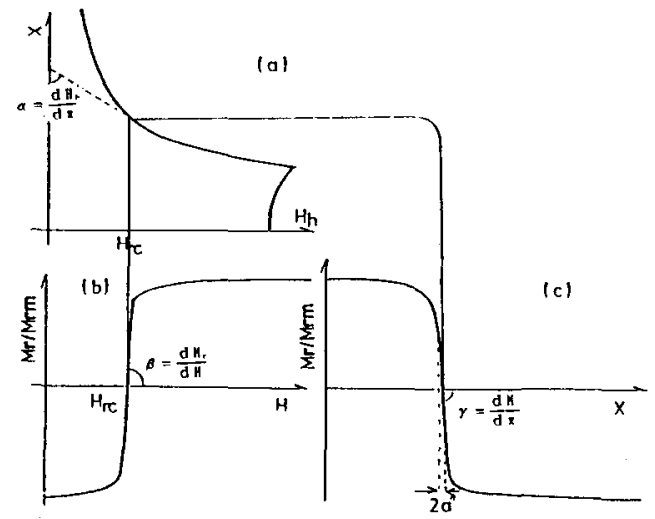

Fig. 1 Inherent magnetization transition obtained without taking demagnetizing field effect into account. 
shown in Fig. 1(b), the center of the transition in the medium is given at the point where the head field is equal to the $\mathrm{H}_{\mathrm{rc}}$ as shown in Fig. 1(a) and (c). So the tangent slope, $\lambda$, at the center of the transition can be calculated as follows,

$$
\begin{aligned}
\left(\frac{\mathrm{d} K_{r}}{\mathrm{dx}}\right)_{H_{h x}=H_{r c}} & =\left(\frac{\mathrm{d} H_{h x}}{\mathrm{dx}}\right)_{H_{h x}=H_{r o}}\left(\frac{d K_{r}}{d H}\right)_{H=H_{r c}} \\
& =\alpha \cdot \beta
\end{aligned}
$$

On the other hand, assuming an arctangent distribution for the magnetization transition, the inherent transition width, $a^{\prime \prime}$, is defined as a distance from the center of the transition to the point where the residual magnetization is a half of the maximum. Therefore, the tangent slope can be also expressed as follows,

$$
\gamma=\left(\frac{\mathrm{d} \mathbf{H}_{\mathrm{r}}}{\mathrm{dx}}\right)_{\mathrm{x}=0}=\frac{2 \mathrm{H}_{\mathrm{r} m}}{\pi \mathrm{a}^{*}}
$$

Consequently, the inherent width, a", can be calculated by the following equation,

$$
a^{*}=\frac{2 M_{r m}}{\alpha \beta \pi}
$$

\section{Actual Magnetization Transition}

In longitudinal magnetic recording, an inherent transition width, a", is widen by the demagnetizing field effect in the transition region. We already reported [4] that an actual magnetization transition width, a, can be graphically and quantitatively calculated with taking the demagnetizing field effect into account.

Now we assume that an inherent transition width is expanded by two steps as shown in Fig. 2. At the first, after the polarity of a head field was reversed, an inherent transition width, $\mathrm{a}^{\prime \prime}$, is widen to an intermediate one, $a^{\prime}$. At the second, after the head moved away, the intermediate transition width, $a^{\prime}$, is more widen to an actual one, a, as shown in Fig. 2(a).

When the inherent width, a", changes to the intermediate one, $\mathrm{a}^{\prime}$, the intensity of magnetization, $M_{r}{ }^{\prime}$, decreases to $M_{r}$ by a demagnetizing field at the point, $X_{1}=a^{\prime \prime}$, as shown Fig. 2(a), according to the following equation,

$$
M_{r}(X 1)=\frac{2}{\pi} M_{r m} \arctan \frac{a^{\circ}}{a^{\circ}}
$$

Here such a magnetization change at the point, $X_{1}$, is given on the residual magnetization curve $I$ shown in Fig. 2(b) in the following section,

$$
H_{r c}\left(H_{r}=0\right) \leq H \leq H_{n}\left(M_{r}=M_{r m} / 2\right)
$$

At the same time, the demagnetizing field strength at the point, $X_{1}$, also decreases according to the following equation,

$$
\begin{aligned}
H_{d}\left(X_{1}\right)=-8 M_{\mathrm{m}} & \left\{\arctan \frac{\mathrm{a}^{\circ}+\delta / 2}{\mathrm{a}^{\circ}}\right. \\
& \left.-\arctan \frac{\mathrm{a}^{\circ}}{\mathrm{a}^{\circ}}\right\}
\end{aligned}
$$

where $\delta$ is the thickness of the recording layer of a medium.

(a)

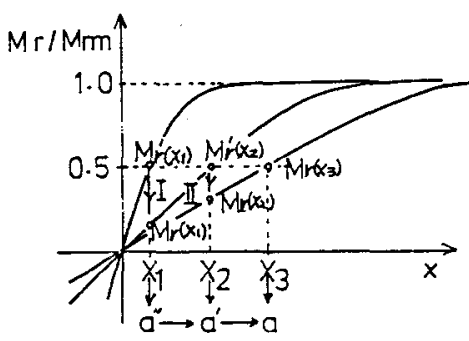

(b)

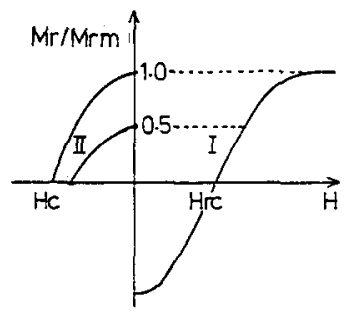

Fig. 2 Two step effects of demagnetizing field on magnetization transition. (a) Expansions of transition width. (b) magnetization curve I and II for each demagnetizing process. 
In the same way, by using the demagnetizing curve II of a $\mathrm{M}-\mathrm{H}$ loop shown in Fig. 2(a) instead of curve $I$, the actual transition width, a, can be obtained by using the same equations as Eq. (4) and (6) and by replacing the $a^{\prime \prime}$ with the $a^{\prime}$, and the $a^{\prime}$ with the a.

Fig. 3 shows the graphical method to obtain an intermediate width, $a^{\prime}$ from an inherent one, $a^{\prime \prime}$, and a final actual width, a, from the intermediate one, $a^{\prime}$. Both heavy solid and broken lines in Fig. 3(a) show the curves I and II of Fig. 2(a). Heavy lines in Fig. 3(b) and (c) show the curves calculated by $\mathrm{Eq} \cdot(4)$ and (6). Using the curve $I$, therefore, the intermediate width, $a^{\prime}$, can be obtained from the value $a^{\prime / \delta}$ which self-consistently gives the magnetization, $M_{r}\left(X_{1}\right)$ at the point, $X_{1}$ $=a^{\prime \prime}$, as shown by thin solid lines linking among these three curves in Fig. 3. The actual transition width, a, can be also obtained from the $a^{\prime}$ by using the curve II in place of the curve $I$.

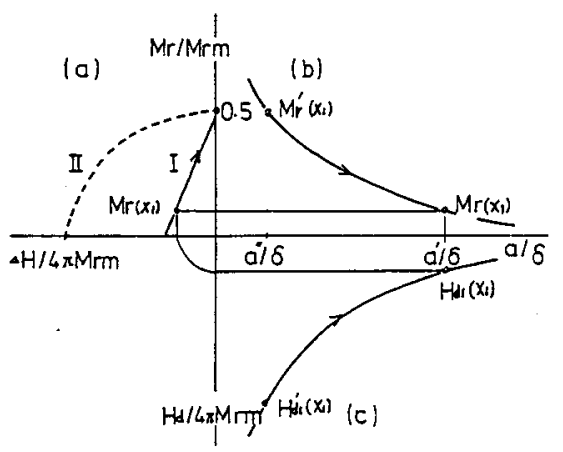

Fig. 3 Graphical method for an actual magnetization transition taking demagnetizing field effect into account.

\section{Reproduced Pulse}

If an actual transition width, a, and the maximum value, $M_{r m}$, of residual magnetization are given, we can restore a magnetization transition in the recording layer by the arctangent distribution as follows,

$$
M_{r}=\frac{2}{\pi} M_{r m} \arctan \frac{X}{a}
$$

Therefore, reproduced voltage can be calculated by the reciprocity using the magneti- zation distribution in the recorded medium and the sensitivity function or the field distribution of a reproducing head.

From the actual transition width, a, moreover, the half-width of isolated pulse, $W_{50}$, can be also calculated by,

$$
W_{5 \theta}=2 \sqrt{\left(a+d_{D}\right)^{2}+\left(g_{\theta}+1 / 2\right)^{2}}
$$

where $d_{p}$ is a head-to-medium spacing and geff is an effective gap length of a reproducing head. When the mean field distribution of a head is taking into account for a thin film medium, the effective spacing, $\mathrm{dy}$, is approximately defined by,

$$
\mathrm{d} r=\mathrm{d}+\delta_{\mathrm{m}} / 2
$$

where $\mathrm{d}$ is the actual spacing and $\delta_{\mathrm{m}}$ is the thickness of the recording layer. And the geff can be given by the half-width of the mean head field distribution.

\section{ISOLATED PULSE}

Perpendicular Recording

In perpendicular magnetic recording, an inherent transition width, a", is equal to an actual transition width, a, because the demagnetizing field hardly arises in a transition region. Hence the actual transition width, a, can be obtained only by the first graphical analysis of Fig. 1.

Solid lines in Fig. 4 show M-H loops of a Co-Cr film measured by a V.S.M. in the direction perpendicular to the surface, and broken lines show those compensating for the influence of the demagnetizing field perpendicular to the medium surface. The

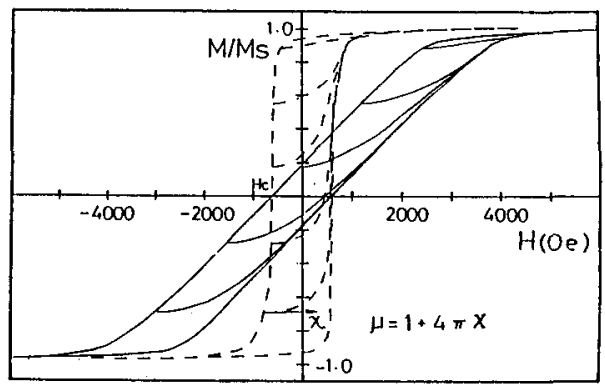

Fig. 4 M-H loop of $\mathrm{Co}-\mathrm{Cr}$ layer. Solid and broken lines show those measured in perpendicular direction and compensated for demagnetizing field. 
compensation has been done so that the slope of the loop at the coercive force becomes infinitive [5]. For the graphical analysis, the actual residual magnetization curve is given from the minor loops of the compensated $\mathrm{M}-\mathrm{H}$ loop.

Fig. 5(a) shows distributions of the perpendicular and longitudinal field components of a single-pole head on the surface of a Co-Cr layer. They were computed by a finite element method (F.E.M.) under the condition as shown in Fig. 5(b). In the F.E.M., the effect of a medium magnetization state on the head field distribution was taking into account by the reversible permeability, $\mu_{\mathrm{y}}$, of the $\mathrm{Co}-\mathrm{Cr}$ layer, because the field distribution strikingly depends on the magnitude of the head-tomedium magnetic interaction [6]. The $\mu_{\mathrm{y}}$ was obtained as an average slope of minor loops at several points reversing from a major to a minor loops shown in Fig. 4. Consequently, as shown in Fig. 5(a), a higher permeability of a $\mathrm{Co}-\mathrm{Cr}$ layer makes a narrower head field distribution having a shaper slope.

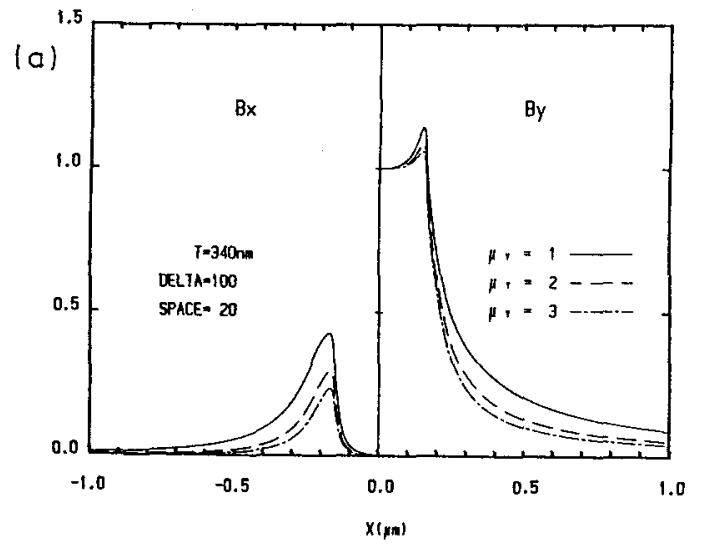

(b)

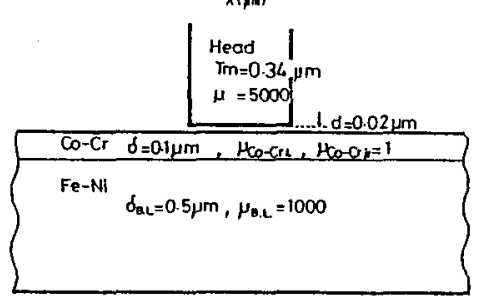

Fig. 5 (a) Field distribution of single-pole head calculated by F.E.M., and (b) calculation condition.
In perpendicular magnetic recording, using a residual magnetization curve and a head field distribution obtained by the methods as shown in Fig. 4 and 5, an inherent transition width, $a^{\prime \prime}$ is graphically obtained as shown in Fig. 1. Then, an isolated pulse is calculated by the reciprocity assuming an arctangent magnetization transition having the width, a", and the head field distribution shown in Fig. 5.

As compared in Fig. 6(a) and (b), the isolated pulses calculated approximately by the graphical method without taking the demagnetizing field effect agree very well with those measured. The measurement has been carried out with a single-pole head and $\mathrm{Co}-\mathrm{Cr} / \mathrm{Ni}-\mathrm{Fe}$ double layered media. The main-pole thickness of the single-pole head is 0.34 microns, and the thicknesses, $\delta$, the perpendicular coercivities, $\mathrm{H}_{\mathrm{c}}$, the saturation magnetization, $M_{S}$ and the reversible permeabilities, $\mu_{\mathrm{y}}$, of each media used are written in the figure.
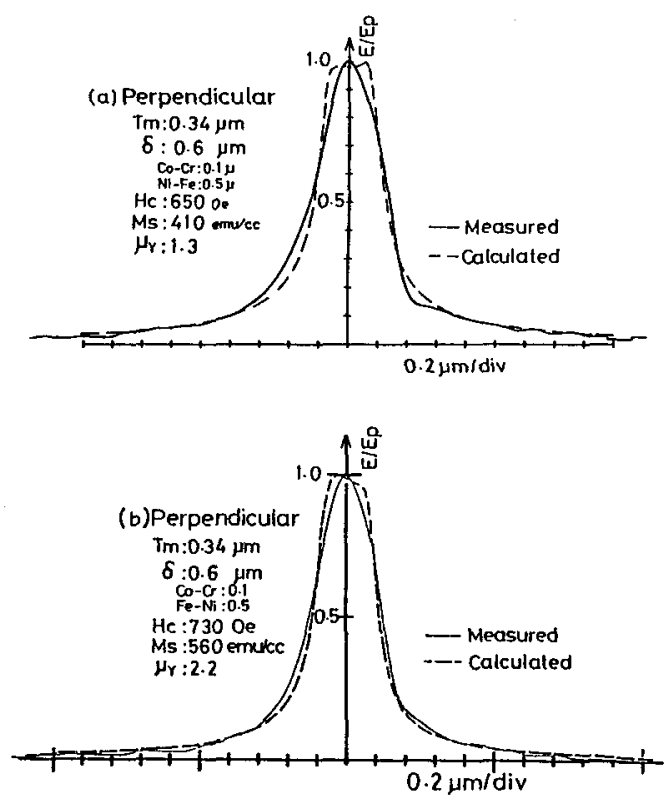

Fig. 6 Comparison of isolated pulses in perpendicular magnetic recording obtained by measurement and graphical analysis.

\section{Longitudinal Recording}

In longitudinal magnetic recording, 
the $\mathrm{a}^{\prime \prime}$ is first obtained graphically using a $\mathrm{M}-\mathrm{H}$ loop of the longitudinal recording medium measured in the longitudinal direction and the mean distribution of the longitudinal component of a ring head field through a recording layer. In Fig. 7, a broken line shows an isolated pulse of a thin film medium calculated by an inherent transition width, a", alone in longitudinal recording. The broken curve obtained without taking the demagnetizing field effect into account differs considerably from the solid curve measured. The measurement has been done by a ring head whose gap length is 0.3 microns. The thickness, $\delta$, the longitudinal coercivity, $H_{C}$, and the saturation magnetization, $\mathrm{M}_{S}$, of a thin metallic tape used were written in the figure.

In order to obtain the actual transition width, a, the inherent width, $a^{\prime \prime}$, has been corrected graphically by the second method as shown in Fig. 3 taking the demagnetizing field effect into account. A dotted-and-dashed line in Fig. 7 shows the isolated pulse calculated from an actual transition width, $a$, which is in very good agreement with the solid line obtained by measurement. Consequently, even for longitudinal magnetic recording, an actual transition width can be graphically obtained in the two steps with taking the demagnetizing field effect into account.

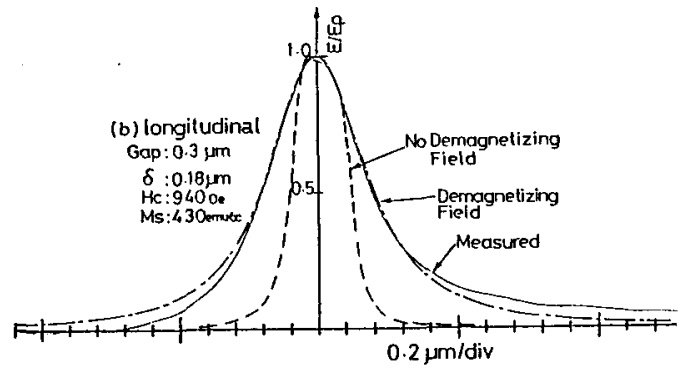

Fig. 7 Comparison of isolated pulses in longitudinal magnetic recording obtained by measurement and graphical analysis.

\section{DISCUSSIONS}

In Fig. 8, the half-pulse widths, W50, measured in both perpendicular and longitudinal recordings are compared with those calculated. White circles show the W50 measured in perpendicular magnetic recording. The measurements has been carried out for various $\mathrm{Co}-\mathrm{Cr} / \mathrm{Ni}-\mathrm{Fe}$ double layer media with a single-pole head having a 0.34 microns thick main-pole. The $\mathrm{Co}-\mathrm{Cr}$ layers are about 0.1 microns in thickness, and have the perpendicular coercivities, $\mathrm{H}_{\mathrm{C}}$, of 650 to $1200 \mathrm{Oe}$, and the saturation magnetization, $M_{S}$, of 320 to $600 \mathrm{emu} / \mathrm{cc}$.

On the other hand, a solid line shows a result calculated graphically. The $W_{50}$ slightly decreases with increasing the reversible permeability, $\mu_{y}$, of the $\mathrm{Co}-\mathrm{Cr}$ layers. A higher permeability results in a stronger magnetization of medium and then a sharper and stronger field distribution of head, because of strong head-to-medium magnetic interaction. The measured values of W50 almost agree with the calculated curve but hardly changes with increasing the $\mu_{\mathrm{y}}$. This is because a $\mathrm{Co}-\mathrm{Cr}$ layer having a higher reversible permeability has a lower squareness in the $\mathrm{M}-\mathrm{H}$ 1oop. As easily expected from the graphical analysis, the lower squareness of a medium results in the wider width of the transition, even if a head field is a sufficiently sharp.

Black triangles in Fig. 8 show $W_{50}$ in longitudinal magnetic recording. They are graphically calculated with taking the demagnetizing field effect into account. White triangles also show $W_{50}$ measured for two thin film media of 0.18 microns and

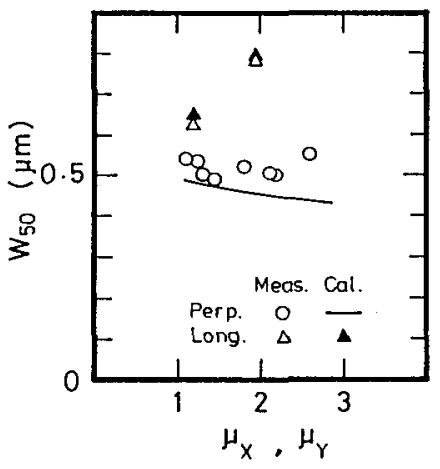

Fig. 8 Comparison of $W_{50}$ calculated and measured for various media having different reversible permeabilities in perpendicular and longitudinal magnetic recordings. 
0.05 microns in thickness. We can confirm that not only the calculated results agree well with the measured ones but also a thinner medium having a lower reversible permeability, $\mu_{x}$, gives a smaller $W_{50}$ because of a higher squareness. However the width of an isolated pulse in longitudinal recording is always wider as compared with those in perpendicular recording by the demagnetizing field effect.

In Fig. 9(a) and (b), the reproduced voltage versus bit density characteristics calculated in perpendicular and longitudinal recordings are shown in comparison with those measured. Broken line with white triangles in Fig. 9(a) shows roll off curves in perpendicular recording, obtained by the superposition of the calculated isolated pulse shown in Fig. 6(b). Solid line with white circles shows relative reproduced voltages measured at various densities. They perfectly coincide up to a high bit density more than $120 \mathrm{kFRPI}$ [7].

On the other hand, solid line in Fig. 9(b) was obtained in longitudinal recording using a metal thin film disk and an un-

(a)

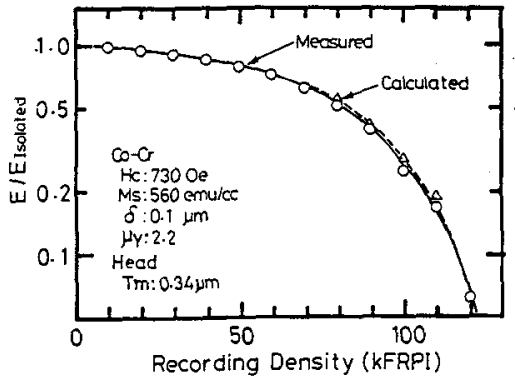

(b)

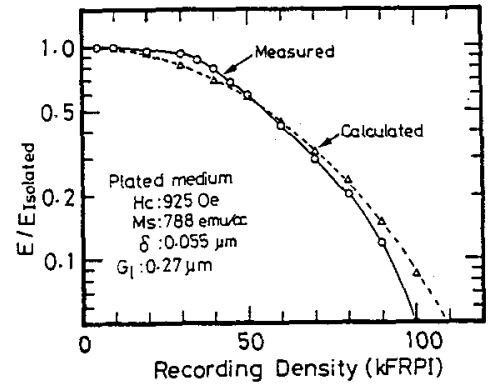

Fig. 9 Comparison of bit density characteristics calculated and measured in (a) perpendicular and (b) longitudinal magnetic recording. floating ring head [8]. Their magnetic properties and gap length are written in the figure. Broken line was calculated by the superposition of an isolated pulse graphically obtained. The difference between both curves clearly increases at higher bit densities because of the demagnetizing field effect in longitudinal magnetic recording.

\section{CONCLUSIONS}

An isolated magnetization transition width in perpendicular magnetic recording is easily and quantitatively estimated by the graphical method without taking the demagnetizing field effect into account. Assuming an arctangent magnetization distribution in the transition, an isolated pulse is restored by the actual transition width and a roll off curve is estimated by the superposition of this pulse. This fact means that perpendicular magnetic recording mechanism is very simple and ideal, while longitudinal recording always includes the demagnetizing field effect.

\section{REFERENCES}

[1] S. Yamamoto, Y. Nakamura and S. Iwasaki : IEEE Trans. Magn, MAG-23, 2071(1987).

[2] Y. Nakamura and I. Tagawa : IEEE Trans. Magn, MAG-24, 2329(19887).

[3] K. Nishimura, Y. Nakamura and S. Iwasaki : Jour. Mag. Soc. Japan, (in Japanese), 13, 141(1989).

[4] Y. Nakamura and S. Iwasaki : Technical Report of IECE Japan, (in Japanese), MR70-19, (1970).

[5] Y. Zhuo, K. Ouchi and S. Iwasaki : Abstracts of 31 st MMM Conf., [AA-07], Nov. (1986).

[6] Y. Nakamura, S. Yamamoto and S. Iwasaki : IEEE Trans. Magn., MAG-22, 376(1986).

[7] S. Iwasaki, Y. Nakamura and H. Muraoka : IEEE Trans. Magn., MAG-17, 2535(1981).

[8] H. Fukuoka and Y. Nakamura : IEICE Technica1 Report, (in Japanese), 89, MR89-4, 1(1989). 